
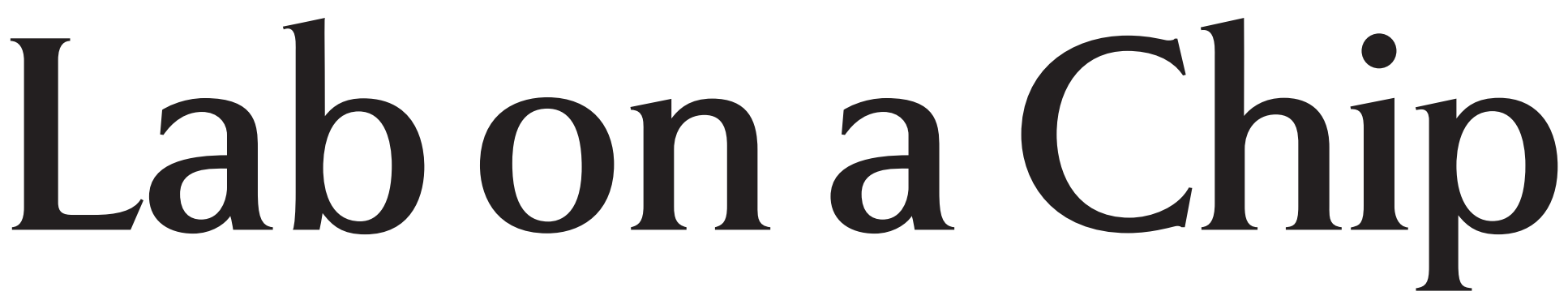

Micro- \& nano- fluidic research for chemistry, physics, biology, \& bioengineering 


\title{
Hybrid opto-electric manipulation in microfluidics-opportunities and challenges
}

\author{
Aloke Kumar, ${ }^{* a}$ Stuart J. Williams, ${ }^{b}$ Han-Sheng Chuang, ${ }^{c}$ Nicolas G. Green ${ }^{d}$ and Steven T. Wereley ${ }^{e}$ \\ Received 8th March 2011, Accepted 20th April 2011 \\ DOI: $10.1039 / c 1 l c 20208 a$
}

\begin{abstract}
Hybrid opto-electric manipulation in microfluidics/nanofluidics refers to a set of methodologies employing optical modulation of electrokinetic schemes to achieve particle or fluid manipulation at the micro- and nano-scale. Over the last decade, a set of methodologies, which differ in their modulation strategy and/or the length scale of operation, have emerged. These techniques offer new opportunities with their dynamic nature, and their ability for parallel operation has created novel applications and devices. Hybrid opto-electric techniques have been utilized to manipulate objects ranging in diversity from millimetre-sized droplets to nano-particles. This review article discusses the underlying principles, applications and future perspectives of various hybrid opto-electric techniques that have emerged over the last decade under a unified umbrella.
\end{abstract}

${ }^{a}$ Biosciences Division, Oak Ridge National Laboratory, Oak Ridge, USA E-mail: kumara1@ornl.gov; Fax: +1 865-574-5345; Tel: +1 865-574-8661 ${ }^{b}$ Department of Mechanical Engineering, University of Louisville, Louisville, USA. E-mail: stuart.williams@louisville.edu; Tel: +1 502-8526340

${ }^{c}$ Mechanical Engineering and Applied Mechanics, University of Pennsylvania, Philadelphia, PA, USA. E-mail: hchuan@seas.upenn.edu; Tel: +1 215-746-2993

${ }^{d}$ School of Electronics and Computer Science, University of Southampton, Southampton, UK. E-mail:ng2@ecs.soton.ac.uk; Tel: +44 (0)2380593778 ${ }^{e}$ Birck Nanotechnology Center, Purdue University, West Lafayette, USA. E-mail: wereley@purdue.edu; Fax: +1 765-496-6443; Tel: +1 765-4945624

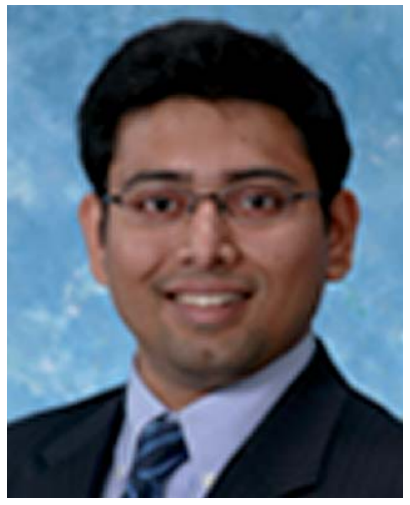

Aloke Kumar
Aloke Kumar received his bachelors and masters degrees from the Indian Institute of Technology, Kharagpur, India in 2005 and his doctoral degree from Purdue University, West Lafayette, USA in 2010. During his doctoral career he was awarded several fellowships, including the prestigious Josephine DeKármán fellowship. Currently, he is a Wigner Fellow and staff member at the Oak Ridge National Laboratory (ORNL). At ORNL his research focuses on phenomena at the microfluidic length scales, particularly lab-on-a-chip electrokinetics and dynamics of microbial aggregations.

\section{Introduction}

The ability to control matter at all length scales, for various applications, has been mankind's relentless goal. The delicate nature of objects involved in lab-on-a-chip devices and microfluidic platforms typically necessitate non-invasive manipulation of matter. Progress in microfluidics and lab-on-a-chip devices in the last few decades saw a concurrent, vigorous activity in the field of non-invasive manipulation of matter and several schemes of manipulation at the micro- and nano-scale have emerged. Today tools exist which employ either light waves, ${ }^{1,2}$ electric fields, ${ }^{3}$ magnetic fields,${ }^{4}$ or acoustic waves ${ }^{5}$ for the non-invasive

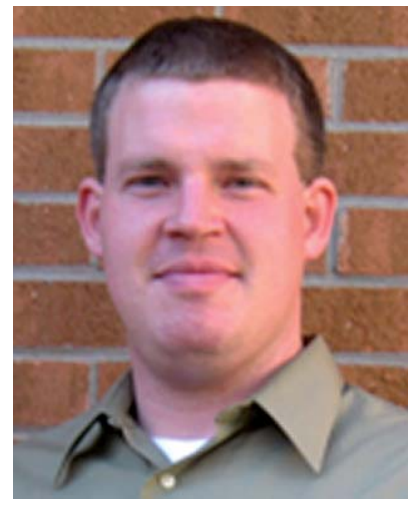

Stuart J. Williams
Stuart J. Williams received his BS and MEng degrees in Mechanical Engineering from the University of Louisville in 2005 and his PhD in Mechanical Engineering from Purdue University in 2009 with the support of the NSF Graduate Research Fellowship. Currently he is an Assistant Professor in the Department of Mechanical Engineering at the University of Louisville where he is the director of the Integrated Microfluidic Systems Laboratory where his research focuses on applications of microfluidics for biology and lab-on-a-chip sample preparation, including optical and electrokinetic methods. 
manipulation of matter at this scale. For example, electric fields generated from patterned electrodes have been employed to move various objects from millimetre-sized aqueous droplets ${ }^{6}$ to nanometre-sized beads. ${ }^{3}$

Innovations in the last decade have resulted in the creation of a new class of non-invasive manipulation techniques-hybrid opto-electric tools. As the name suggests, these techniques employ simultaneous light waves and electric fields to achieve fluid or particle manipulation at the micro- and nano-scale. Unlike, optical tweezing, ${ }^{7}$ hybrid tools do not rely on optical pressure for non-invasive manipulation. Instead, these techniques use light to activate and control electrokinetic manipulation mechanisms. The simultaneous presence of light and electric fields, and their non-linear interaction can result in the appearance of physical phenomena unique to opto-electric systems compared to traditional electrokinetic systems. The use of light also bestows on these techniques a dynamic nature and

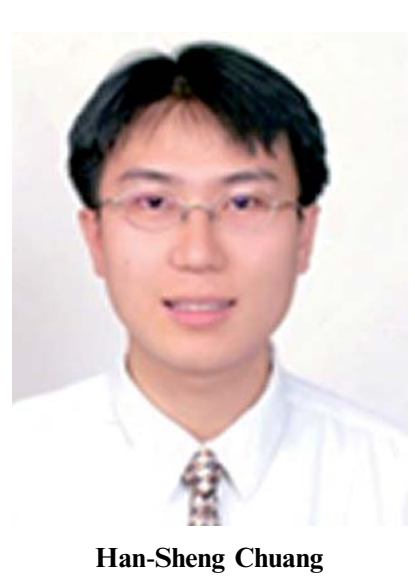

Han-Sheng Chuang

Han-Sheng Chuang received his PhD degree in department of mechanical engineering from Purdue University, West Lafayette, IN in 2010. He is currently a postdoctoral researcher working with $\mathrm{Dr}$ Haim Bau at University of Pennsylvania. His research interests are focused on microl nano-fluidics, MEMS/NEMS technologies and optical diagnostics. In 2005, he was awarded a competitive fellowship from Ministry of Education in Taiwan. He and his research fellows were also the finalists of the prestigious Burton D. Morgan Business Competition in 2008 and 2009, respectively. In addition, he is a co-founder of a US based technical start-up, Microfluidic Innovations, founded in 2009.

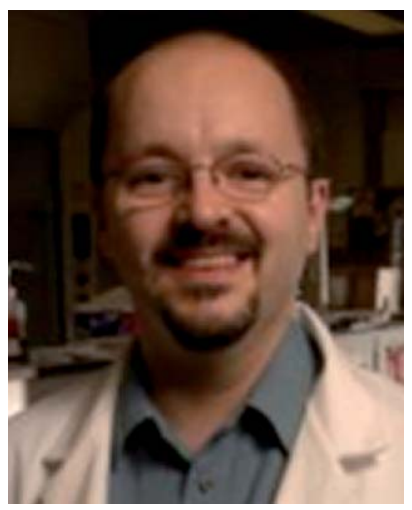

Nicolas G. Green

Nicolas Green is a Reader in the Nano Group and the Southampton Nanofabrication Centre in ECS at the University of Southampton. He studied for a PhD from University of Glasgow from 1994 until 1997 and then worked as a Marie Curie Research Fellow at the University of Sevilla, Spain and a Royal Academy of Engineering Fellow at the Universities of Glasgow and Southampton. He was appointed to a lectureship at the University of Southampton in 2005. His research interests are in the areas of Bioelectronics and Microfluidics examining fundamental physics and applications of electrokinetics in lab-on-a-chip systems. a higher degree of freedom. This freedom enables reconfigurable and programmable lab-on-a-chip devices driven through illumination displays such as a LCD screen or simply with a laser pointer. A variety of optically induced electrokinetic techniques spanning a range of length scales have emerged.

A structured discussion on the diverse opto-electric techniques is facilitated by a classification based on the operational length scale $(l)$ of the technique. Attempting such a classification, one finds that the operational length scales can be divided into two broad groups. One class comprises of techniques that manipulate liquid-suspended particulates whose dimensions range from nanometres to micrometres i.e. $l \approx 10^{-9}$ to $10^{-6} \mathrm{~m}$. Techniques such as optoelectronic tweezers ${ }^{8}$ (OET) and rapid electrokinetic patterning 9 (REP) would belong to this class. Another class consists of techniques dealing with object dimensions in the millimetre scale i.e. $l \approx 10^{-4}$ to $10^{-3} \mathrm{~m}$. Optoelectrowetting ${ }^{10}$ (OEW) and its derivatives such as open optoelectrowetting $\left(\mathrm{O}-\mathrm{OEW}{ }^{11}\right)$ and single-sided continuous optoelectrowetting $\left(\mathrm{SCOEW}^{\mathbf{1 2}}\right)$ comprise this class of hybrid techniques. Unlike REP or OET, OEW and its derivatives deal with the controlled actuation of droplets. Multi-scale manipulation using a combination of different techniques is also possible and will be discussed here. Fig. 1 depicts a chart of the different hybrid optoelectric techniques that have emerged over the past decade.

Such hybrid techniques are engendering new applications in lab-on-a-chip systems, and are enabling novel approaches to the investigation and application of fundamental electrokinetic phenomena. Given the recent emergence of these techniques, this article will discuss the various technologies that have been proposed in the last decade under the unified umbrella of hybrid opto-electric techniques. We start with a discussion of techniques, whose typical operational length scales lie between $l \approx$ $10^{-9}$ and $10^{-6} \mathrm{~m}$, subsequently we will discuss techniques with $l \approx$ $10^{-4}$ to $10^{-3} \mathrm{~m}$. We will conclude by discussing current challenges and opportunities for continued research in hybrid techniques.

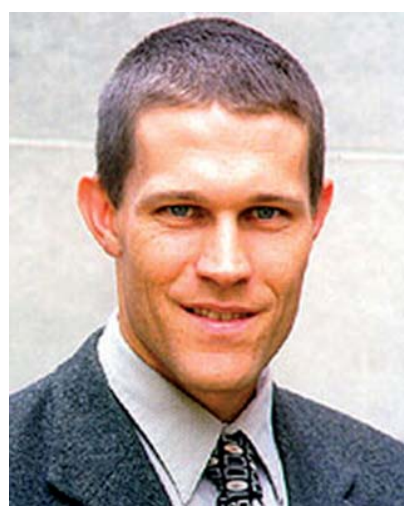

Steven T. Wereley
Professor Wereley has bachelor degrees in Physics (Lawrence University, 1990) and Mechanical Engineering (Washington University, St Louis, 1990) followed by masters and doctoral degrees from Northwestern University (1992 and 1997). He is currently Professor of Mechanical Engineering at Purdue University and a Fellow at the Center of Smart Interfaces, Technische Universität Darmstadt (Germany). Professor Wereley served as an Alexander von Humboldt Fellow at Technische Universität Darmstadt (2007) and Universität der Bundeswehr (2009). Professor Wereley is the co-author of the monographs Fundamentals and Applications of Microfluidics (Artech House, 2002 and 2006) and Particle Image Velocimetry: A Practical Guide (Springer, 2007). 


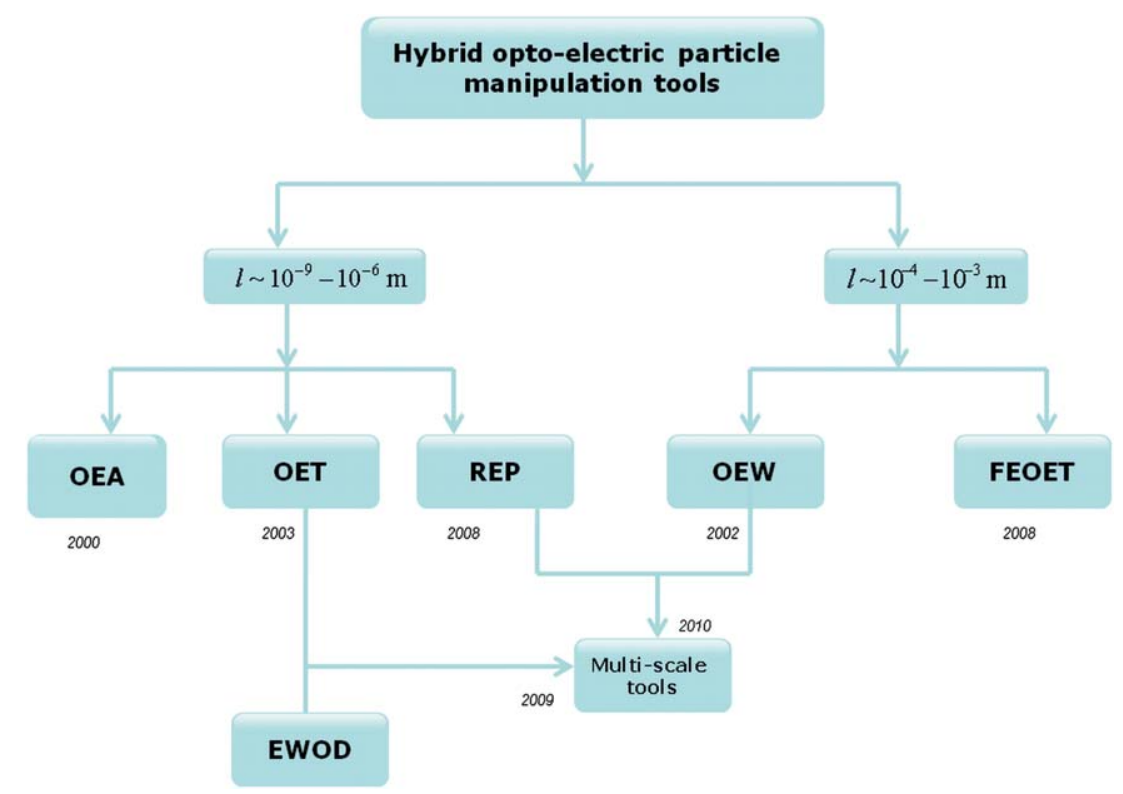

Fig. 1 A chart classifying newly developed opto-electric techniques. The text below the boxes indicates the year in which the technique was first reported in the literature.

\section{Hybrid techniques with $l \approx 10^{-9}$ to $10^{-6} \mathrm{~m}$}

There are primarily three techniques that fall within this category viz., opto-electrohydrodynamic assembly (OEA), OET and REP. Not only are the original implementations of these three techniques very different, the underlying physical principles and the abilities are also distinct. Table 1 provides a quick overview of these techniques before we proceed on an individual discussion of each.

\subsection{Opto-electrohydrodynamic assembly}

One of the first innovations in the field of hybrid opto-electric techniques was led by Hayward et al. ${ }^{13}$ in 2000 . They used optical patterns shone on an electrode surface to assemble suspended particles into optically tunable crystals. The electrokinetic setup consisted of a parallel plate configuration with a brass cathode and an indium tin oxide (ITO) thin film as the anode. Ultra-violet (UV) light was first passed through a pre-fabricated mask and the resulting pattern was illuminated on the ITO film. The microchannel was filled with a dilute aqueous solution of polystyrene (PS) beads. Hayward et al. ${ }^{13}$ observed that over time, the PS beads aggregated on the ITO surface, under the influence of an applied DC field, and the aggregation resulted in dense packed assembles in patterns similar to the pattern of UV irradiation (Fig. 2a). Hayward et al.' 's ${ }^{13}$ process of assembling such optically tunable crystals took several hours and the researchers proposed that the illuminated regions on the ITO surface had a higher current density than the dark regions. Following the pioneering work of Hayward et al., ${ }^{13}$ Gong and $\mathrm{Marr}^{14}$ demonstrated rapid crystal formation by the use of AC signal biased ITO electrodes and laser $(532 \mathrm{~nm})$ scanned patterns. Just as in the case of Hayward et al. ${ }^{13}$ the assembled crystals resembled the shape of the laser scans. Gong and Marr $^{14}$ showed the existence of polycrystallinity in the assembled structures, and that not only can nucleation be directed with control over crystal annealing and melting.

The basis of the reported colloidal aggregation under the influence of UV light is not fully understood, but it is believed to be related to planar particle aggregation on electrode surfaces
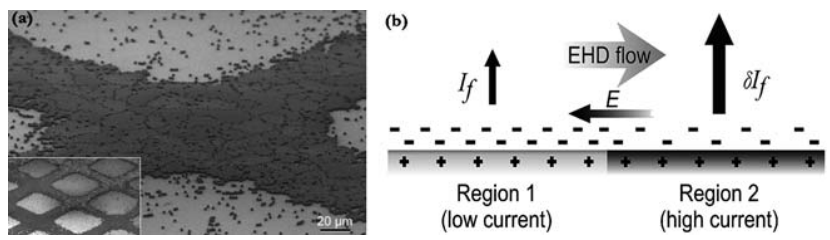

Fig. 2 (a) The figure shows the assembly of PS beads on ITO surface using optical motifs, as outlined in Hayward et al. ${ }^{13}$ Reprinted with permission from Macmillan Publishers Ltd: Nature, Hayward et al., ${ }^{13}$ copyright 2000. (b) Non-uniform currents drive flow, which in turn can entrain particles leading to an aggregation. Reprinted with permission from Ristenpart et al. ${ }^{33}$ Copyright 2008 American Chemical Society.

Table 1 Table listing the predominant hybrid techniques with $l \approx 10^{-9}$ to $10^{-6} \mathrm{~m}$

\begin{tabular}{llll}
\hline Technique & Original setup & Distinguishing feature & $\begin{array}{l}\text { Notable deviations } \\
\text { from initial setup }\end{array}$ \\
\hline OEA & Parallel plate, UV light source with static mask (ref. 13) & Use of non-uniform currents & Ref. 14 \\
OET & Use of a-Si, DMD with near-red light emitting diode laser (ref. 8) & $\begin{array}{l}\text { Use of virtual electrodes } \\
\text { Use of optically driven electrothermal flows }\end{array}$ & $\begin{array}{l}\text { Ref. 70-42 and 92 } \\
\text { REP }\end{array}$ \\
\hline
\end{tabular}


due to the action of both DC and AC fields. ${ }^{15-18}$ The latter phenomena first reported by Richetti et al., ${ }^{16}$ were modeled by Trau et al. ${ }^{17,18}$ as a perturbation of the local electric field by particles and the subsequent action of the same perturbations on the electrode polarization layer. This model proposed by Trau et al. ${ }^{18}$ is actually an instance of induced-charge electro-osmosis (ICEO) flows. ${ }^{19-21}$ Later, Ristenpart et al. ${ }^{22}$ provided scaling laws for such flows with $\mathrm{AC}$ frequency $(\sim 1 / \omega)$ and AC field strength $\left(\sim|E|^{2}\right)$. However, the inverse dependence on AC frequency leads to unrealistic predictions in the limit of low AC frequencies $(\omega \rightarrow$ $\left.0^{+}\right)$. For steady or DC fields, Solomentsev et al. ${ }^{23,24}$ proposed alternate mechanisms based on electro-osmotic slip flows (EOFs) on the particles' surface with no polarization change. Each model has its own drawbacks and a possibility is that a combination of models can satisfactorily explain the diversity of observed phenomena. In fact a recent investigation by Ristenpart et al. ${ }^{25}$ has indicated that both Trau et al.' ${ }^{18}$ and Solomentsev et al.' ${ }^{23,24}$ model need to be invoked in order to explain aggregation in steady fields. A complete review of aggregation on electrodes and effects of various inhomogeneities is outside the scope of this review, but we would like to note that several researchers have reported significant findings and readers might wish to refer to the some of the following reports. ${ }^{26-35}$ Hayward et al.'s ${ }^{13}$ use of optical motifs is believed to induce higher ionic current densities in regions adjacent to the illumination prompting flows of the above described nature, which in turn entrains the particles and ultimately leads to an aggregation resembling the optical motif $^{13,33}$ (Fig. 2b).

\subsection{Optoelectronic tweezers}

Illustrated in the previous section, Hayward et al. ${ }^{13}$ laid the foundations of electric field perturbations by local increase of conductivity of a substrate. Chiou et al. ${ }^{\mathbf{8} 36}$ explored materials whose photoconductivity lay in a different regime. By the use of a highly efficient photoconductive material deposited on a planar surface along with a programmable illumination system, Chiou et $a l .{ }^{8,36}$ demonstrated that illuminated areas generate 'virtual' electrodes, which are dynamically reconfigurable to electrokinetically manipulate particles and fluid. This tool called optoelectronic tweezers (OET) made possible the use of standard electrokinetic techniques without the use of patterned electrodes.

The operation of OETs was demonstrated by Chiou et al. ${ }^{8}$ and is illustrated in Fig. 3. This device consisted of unpatterned parallel ITO electrodes with one of them being coated with hydrogenated amorphous silicon (a-Si:H) serving as the photoconductive material. Amorphous silicon is a widely used material in the solar cell industry; when light illuminates a-Si:H its electrical conductivity increases several orders of magnitude, depending on the illumination intensity. ${ }^{37}$ The increased conductivity will lead to a localized increase in field strength at the substrate surface, enabling dynamic virtual electrode configurations. OET can be activated with a white light source, although a-Si:H absorption is most efficient from red to nearinfrared wavelengths. ${ }^{38}$

The types of illumination sources and its delivery to the photoconductive surfaces vary. A static illumination pattern can be simply applied by the use of a single laser spot ${ }^{39}$ or a photomask. ${ }^{13}$ However, OET systems are characterized by their ability to dynamically reconfigure light patterns. These dynamic systems are driven by a digital micromirror device (DMD), ${ }^{8,39}$ liquid crystal display (LCD) ${ }^{40,41}$ or a beam projector. ${ }^{42}$ These systems require the integration of optical components to focus the illumination on a microscope stage and provide a resolution of 1-20 $\mu \mathrm{m}$. However, an LCD-based system without integrated optics has been demonstrated that grants more portability at the cost of increased electrode resolution $(200 \mu \mathrm{m}){ }^{40}$ Regardless of the illumination scheme, OET illumination intensity is over 100000 times less than traditional optical tweezing systems and has a much larger working area. ${ }^{8}$

The electrode configuration for a traditional OET device is similar to of Fig. 3, which illustrates a closed system with the photoconductive material on one side with particle manipulation occurring on the photoconductive substrate surface. However, a three-dimensional manipulation scheme has been demonstrated with photoconductive materials on both the top and bottom electrodes. ${ }^{43}$ Single-sided OET devices have also been developed with a single ITO substrate patterned with interdigitated electrodes and coated with a-Si:H. ${ }^{44}$ The limitation of using a simple a-Si:H film is the restriction of particle manipulation in low conductivity $\left(<100 \mathrm{mS} \mathrm{m}^{-1}\right)$ solutions; biological media like phosphate-buffered saline (PBS) and Dulbecco's Modified Eagle Medium (DMEM) have conductivities from 1 to $2 \mathrm{~S} \mathrm{~m}^{-1} \cdot{ }^{45}$ To overcome this issue, phototransistors were incorporated with an OET system and the system demonstrated particle manipulation with high conductivity media $\left(>1 \mathrm{~S} \mathrm{~m}^{-1}\right){ }^{46,47}$ This capability, though, comes with the added expense of fabricating complex structures instead of the traditional a-Si:H film.

OETs can generate a variety of electrokinetic particle and hydrodynamic forces. ${ }^{48}$ Dielectrophoresis (DEP) is the most widely used electrokinetic mechanism in OETs. DEP refers to the translation of polarizable neutral particles in the presence of a non-uniform electric field, typically an AC field. ${ }^{3}$ The magnitude and direction of the DEP force are proportional to the volume of the particle, the signal voltage and frequency, and the gradient of the applied field; it's also a function of the dielectric properties (permittivity, conductivity) of the fluid and particle. DEP forces are categorized as positive, being attracted to field gradients, or negative, being repelled from these regions. With regards to OETs, particles are attracted to illuminated regions under positive DEP but are repelled from them with negative DEP. Fig. $4 a$ and $b$ provide examples of particle trapping using both types of DEP forces.

When a particle is polarized the two oppositely charged poles are aligned in the direction of the electric field. If a non-spherical particle undergoes polarization, the poles will tend to concentrate at the ends of the long axis of the particle. This will induce a torque force on the particle to orient its long axis with the direction of the electric field. This phenomenon is called electroorientation and can be used to extract dielectric properties from the particle. ${ }^{4}$ OET devices have applied this technique to align nanowires ${ }^{49}$ and bacteria. ${ }^{50}$ Particle's charged poles will also interact with neighboring particles. Similarly charged particles will form 'chains' and align in the direction of the electric field. However, if adjacent particles are perpendicular to the direction of the electric field they are repelled from each other. These particle-particle interactions have also been observed in an OET device. ${ }^{51}$ 


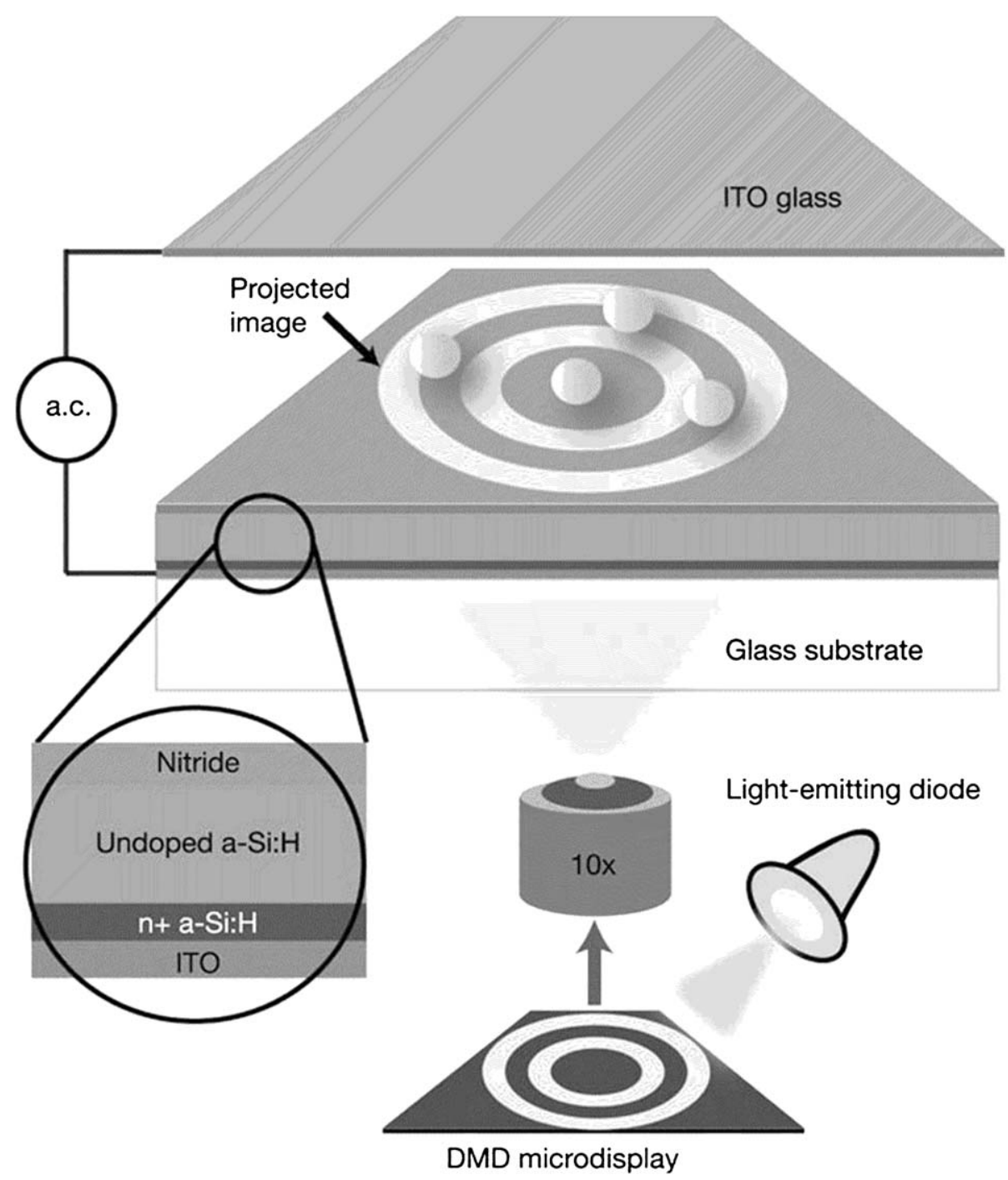

Fig. 3 Illustration of a typical OET platform characterized by a photoconductive film (a-Si:H) and a projection of dynamic light patters, in this case from a DMD. Reprinted with permission from Macmillan Publishers Ltd: Nature, Chiou et al. ${ }^{8}{ }^{\text {copyright }} 2005$.

OETs can also induce two electrohydrodynamic mechanisms, AC electroosmosis (ACEO) and electrothermal (ETH) motion. ACEO is induced when the tangential component of the electric field moves ions within the substrate's electric double layer, generating a slip velocity. ${ }^{52}$ This frequency-dependent behavior is dominant at low frequencies $(<10 \mathrm{kHz})$ and at large Debye lengths (i.e. low conductivity media). ACEO is particularly useful in the trapping of sub-micrometre particles where Brownian motion overcomes DEP forces. In OETs, the illuminated area can generate ACEO flows, carrying suspended particles towards these regions. Such flows have captured particles ranging from $50 \mathrm{~nm}$ to $2 \mu \mathrm{m} .{ }^{39}$ Selective and rapid concentration of $1 \mu \mathrm{m}$ and $6 \mu \mathrm{m}$ polystyrene particles was demonstrated utilizing simultaneously applied ACEO and DEP. ${ }^{53}$ ETH hydrodynamics occur when the applied electric field acts upon fluid dielectric inhomogeneities resulting from non-uniform temperature fields. ${ }^{9}$ Such temperature gradients can be generated through Joule heating or with applied external illumination. ${ }^{54}$ The photoconductive material in OETs absorb the applied illumination resulting in ETH flows, but such flows generally overcome other electrokinetic forces only at illuminations greater than for typical OET use. ${ }^{48}$ Jamshidi, et al. ${ }^{55}$ demonstrated aggregation of $90 \mathrm{~nm}$ diameter gold nanoparticles with OET-induced ETH flows. Fig. $4 \mathrm{c}$ provides an example of particle manipulation using ACEO and ETH electrohydrodynamics driven by OETs.

OETs in its various forms has used electrokinetic mechanisms for trapping, patterning, and sorting a variety of particles. Manipulated non-biological particles outside of traditional polystyrene/silica beads include quantum dots, ${ }^{39}$ silver nanorods ${ }^{49}$ gold nanoparticles, ${ }^{55}$ and carbon nanotubes ${ }^{56}$ Biological sorting demonstrations with OET include motile and nonmotile sperm, ${ }^{57}$ erythrocytes and leukocytes, ${ }^{58}$ live and dead human B cells, ${ }^{8}$ and normal and abnormal oocytes. ${ }^{59}$ Additional biological entities that have been manipulated with OET include HeLa cells, ${ }^{60}$ E. coli,${ }^{61}$ yeast, ${ }^{42}$ protozoa, ${ }^{39}$ DNA, ${ }^{62}$ and HepG2 cells. $^{63}$ To reduce non-specific adhesion an operational OET device coated with poly (ethylene glycol) was demonstrated. ${ }^{64}$ For an expanded discussion on the applications of OET, readers are referred to a recent review article by Hwang and Park. ${ }^{65}$ 
(a)
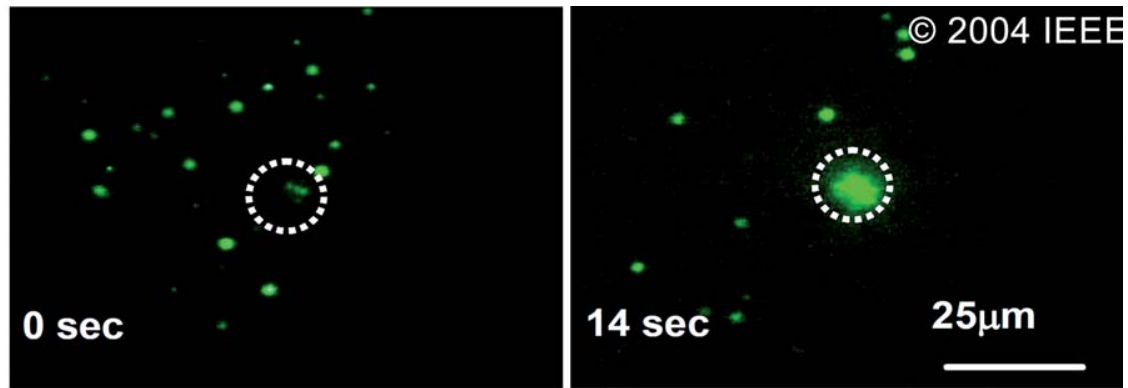

(b)
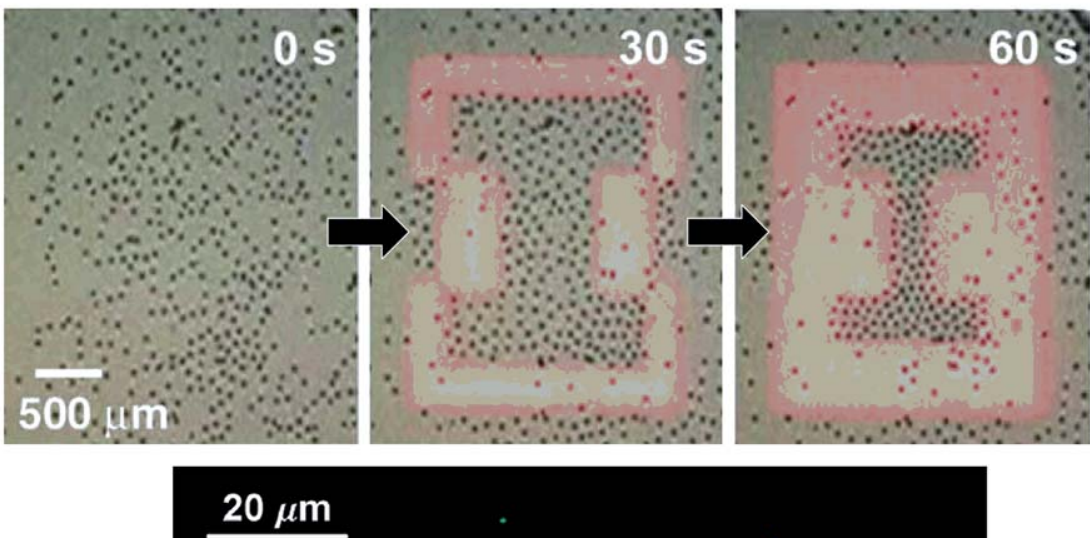

(c)

\section{$2 \mathrm{~s} 10 \mathrm{~s} 20 \mathrm{~s} \quad 30 \mathrm{~s} \quad 60 \mathrm{~s} \quad 120 \mathrm{~s}$}

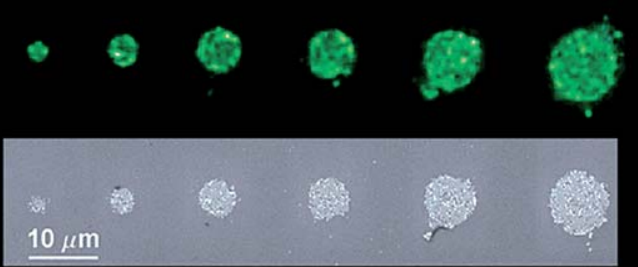

Fig. 4 (a) Positive DEP trapping of E. coli with OETs is demonstrated when they are attracted to an illuminated 17 mm spot-size. From Chiou et al. ${ }^{61}$ Reproduced with permission of IEEE, (2004 IEEE. (b) $45 \mathrm{~mm}$ particles are aggregated in dark regions of the dynamic illuminated pattern with negative DEP. From Choi et al. ${ }^{40}$ Reproduced with kind permission of Springer Science + Business Media. (c) Trapping of $90 \mathrm{~nm}$ gold nanoparticles with a combination of ACEO and ETH hydrodynamics. Reprinted with permission from Jamshidi et al. ${ }^{55}$ Copyright 2009 American Chemical Society.

\subsection{Rapid electrokinetic patterning}

Rapid electrokinetic patterning (REP) is a more recent hybrid opto-electric technique introduced by Williams et al. ${ }^{9}$ in 2008 as a real time dynamic particle manipulation technique. The original chip configuration utilized by Williams et al. ${ }^{9}$ consisted of ITO coated parallel electrodes biased with an AC signal (Fig. 5). Williams et al. $^{9}$ showed that in a low frequency regime $(<200$ $\mathrm{kHz}$ ), micrometre dimension particles could be trapped and dynamically configured into various shapes on the electrode surface by using various optical landscapes produced from a laser operating at a wavelength of $1064 \mathrm{~nm}$. REP is a versatile technique and particles as diverse as gold particles $(200,250 \mathrm{~nm})$ (unpublished data), silica particles, ${ }^{66}$ and polystyrene particles (50 $\mathrm{nm}$ (ref. 67) to $3 \mu \mathrm{m}$ ) have been successfully aggregated. A video demonstrating REP and highlighting its features is available. ${ }^{68,69}$

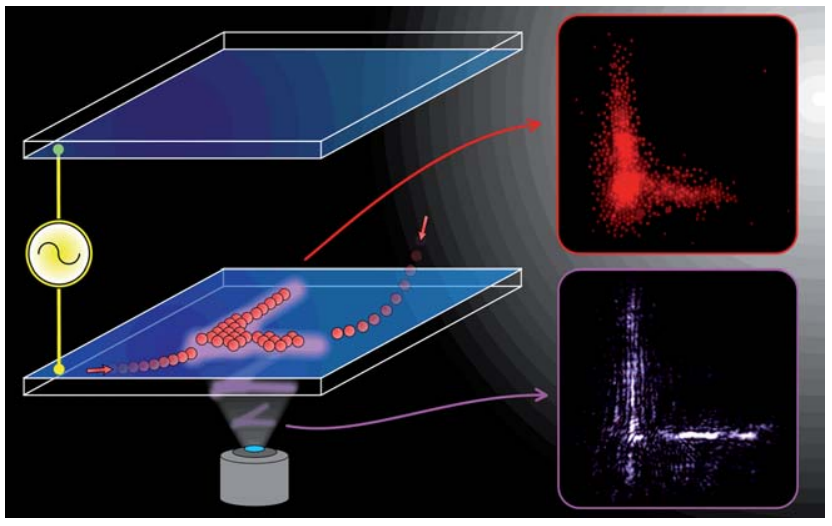

Fig. 5 An example of the REP manipulation technique. An intense laser hologram is shone on an ITO surface and the $690 \mathrm{~nm}$ red fluorescent PS beads take the shape of the hologram. Williams et al. ${ }^{9}$ Reproduced by permission of The Royal Society of Chemistry. 
No single electrokinetic mechanism drives REP-based particle aggregation and research has shown that it is actually brought about by the concerted action of a variety of electrokinetic forces. An understanding of the REP process can be facilitated by seeking answers to these distinct questions: (i) What causes rapid particle transport to illuminated regions on the electrode surface? (ii) What keeps the particles on the electrode surface? (iii) What is the structure of the aggregated particles? While these questions are interrelated, the following will approach these questions individually. First to be addressed will be the driving forces for particle transport to the illuminated regions. Kumar et al. ${ }^{70}$ demonstrated that a highly focused $1064 \mathrm{~nm}$ laser beam shone on an ITO surface can cause highly localized temperature gradients by the heating of the substrate (Fig. 6a). For a laser power of $20 \mathrm{~mW}$ the absolute temperature change itself was not very significant $(\sim 5 \mathrm{~K})$, but this change occurred at the length scale of microns, resulting in very high temperature gradients $\left(\sim 10^{5} \mathrm{~K} \mathrm{~m}^{-1}\right)$. Such high temperature gradients in the presence of electric fields can generate ETH body forces ${ }^{3,54,71}$ and thus result in dominant ETH flows. In another work, Kumar et al. ${ }^{72}$ demonstrated that a three-dimensional electrothermal microvortex (EMV) formed within an REP device, which has a 'sinktype' behavior near the electrode surface. Using two-dimensional micron resolution particle image velocimetry ( $\mu$ PIV) they showed that the strength of the microvortex varies as the square of the electric field strength, $|E|^{2}$, consistent with electrothermal hydrodynamic theory. Later, Kumar et al. ${ }^{73}$ used a novel threedimensional wavefront deformation particle tracking velocimetry (PTV) developed at Universität der Bundeswehr München ${ }^{74}$ to obtain a three-dimensional velocity map of the EMV (Fig. 6b). They also showed good agreement between experimental data and numerical simulations, which were based on ETH body force calculations. Williams et al. ${ }^{9}$ conjectured that the EMV transports particles from bulk to the electrode surface, where they are 'captured'. Williams et al. ${ }^{66}$ characterized colloidal accumulation in REP as a function of time and the imposed electric field, and found that the rate of colloidal accumulation varied as the square of the electric field strength, in agreement with the hypothesis. They went on to show that a $532 \mathrm{~nm}$ laser can be used in conjunction with a gold electrode to produce particle aggregations, similar to those demonstrated with an ITO electrode using $1064 \mathrm{~nm}$ laser beam. This feature is significant as it allows REP to be replicated at different optical wavelengths, provided that the proper combination of electrode-laser wavelength is chosen to generate the necessary optically induced temperature gradients.

Once transported to the illuminated site(s), particle-electrode interaction forces allow the particles to be 'trapped'. As the drag from the toroidal EMV would tend to 'pluck' the transported particles away from the electrodes, the particle-electrode interaction forces are attractive in the REP regime, but are a function of frequency, medium conductivity and possibly even the electrolytic species, but are expected to be independent of illumination. Fagan et al.' $\mathrm{s}^{75-77}$ work comprises the very select studies that have concentrated their efforts on localized particle-electrode forces. By measuring the temporal variations in a particle's height above an electrode $(h)$, using total internal reflection microscopy, they concluded that the observed oscillations in $h$ could only be explained through the superposition of different mechanisms. Most significantly they concluded that the electrohydrodynamic flows produce a vertical force component on the particle for frequencies above $500 \mathrm{~Hz}$. Apart from forces exerted by the fluid, other mechanisms can also contribute to these vertical forces on the colloidal particles. Image charges can play a role in the particle-electrode interaction. ${ }^{78,79}$ It has also been shown that bringing two electrical double layers (EDLs) in close proximity can create attractive or repulsive forces. ${ }^{80-82}$ This explains the second question, though a complete characterization of the particle-electrode interaction forces remains elusive. However, one can conceive the use of the EMV vortex itself to measure these net attractive forces.

The structure of particle aggregations in a REP cluster has not been extensively investigated. Typically, larger particles $(>1 \mu \mathrm{m})$ tend to form a monolayer, smaller particles $(<300 \mathrm{~nm})$ tend to exhibit a multi-layer characteristic. ${ }^{67}$ It is possible that oscillatory behavior of particles near the electrode ${ }^{75-77}$ has an influence on the aggregation structure.

In its very short span of development, the REP technique has shown to lend itself to a variety of different applications. Kumar et al. ${ }^{70}$ proposed a new force spectroscopy technique based on the

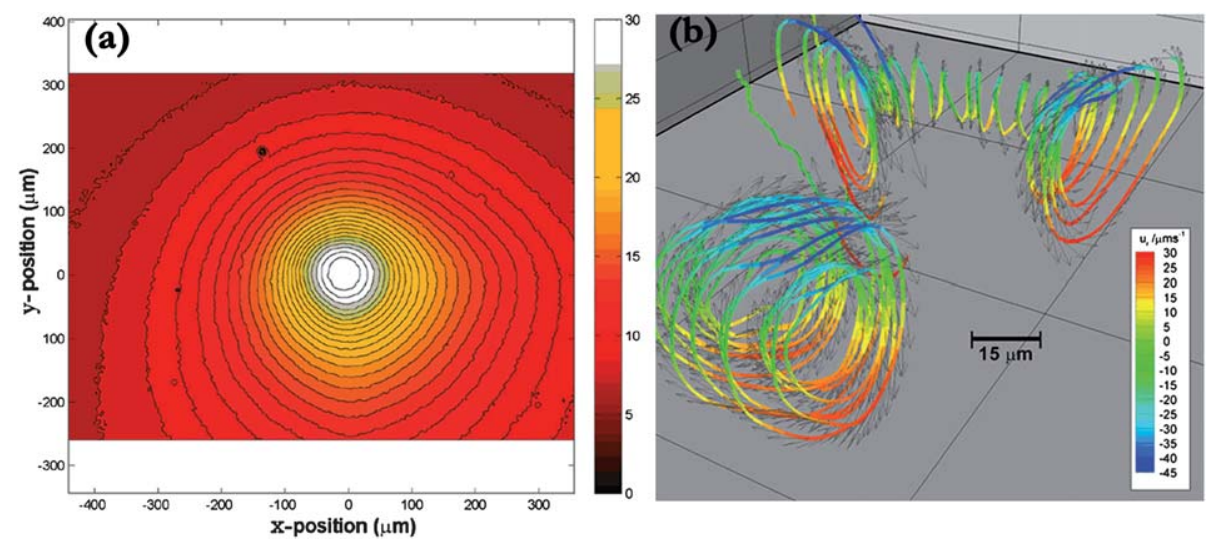

Fig. 6 (a) The $1064 \mathrm{~nm}$ laser causes intense temperature gradients on the ITO surface. Reprinted with permission from Kumar et al. ${ }^{70}$ Copyright 2010 American Chemical Society. (b) The EMV as visualized using wavefront deformation PTV. The EMV transports analytes from the bulk to the electrode surface. From Kumar et al..$^{73}$ Reproduced with kind permission from Springer Science + Business Media. 
REP technique. They used REP to capture an aggregation of $1 \mu \mathrm{m}$ particles, and subsequently deactivated the laser. In the absence of the laser heating, the electrothermal flow ceases, allowing the particle-cluster to 'explode' due to inter-particle repulsive forces (Fig. 7). Using Delaunay triangulation, Kumar et $a .^{70}$ defined an ensemble-averaged inter-particle distance, whose time rate of increase provides a measure of the average inter-particle repulsive force. Repeating the procedure for various $\mathrm{AC}$ frequencies and voltages, Kumar et al. $^{70}$ found an anomalous decrease in the dipole-moment of the particles. This anomalous behavior was accounted for on the basis of an additional dipole moment contribution from a non-equilibrium EDL. ${ }^{83-86}$ This phenomenon arises from the polarization of the EDL, around a particle, in the presence of an electric field. ${ }^{79,85-88}$ Kumar et $a l^{70}$ concluded that the dipole moment of the particle in the REP regime is given the combination of the MaxwellWagner interfacial polarization ${ }^{3}$ and non-equilibrium EDL polarization.

An application of REP for sorting of colloids was demonstrated by Williams et al. ${ }^{66}$ Williams et al. ${ }^{66}$ were able to sort PS beads of sizes $0.5,1$ and $2 \mu \mathrm{m}$ by varying the AC frequency between 38 and $106 \mathrm{kHz}$ (Fig. 8). Williams et al. ${ }^{66}$ also demonstrated sorting between $1 \mu \mathrm{m}$ PS and $1 \mu \mathrm{m}$ silica beads by varying
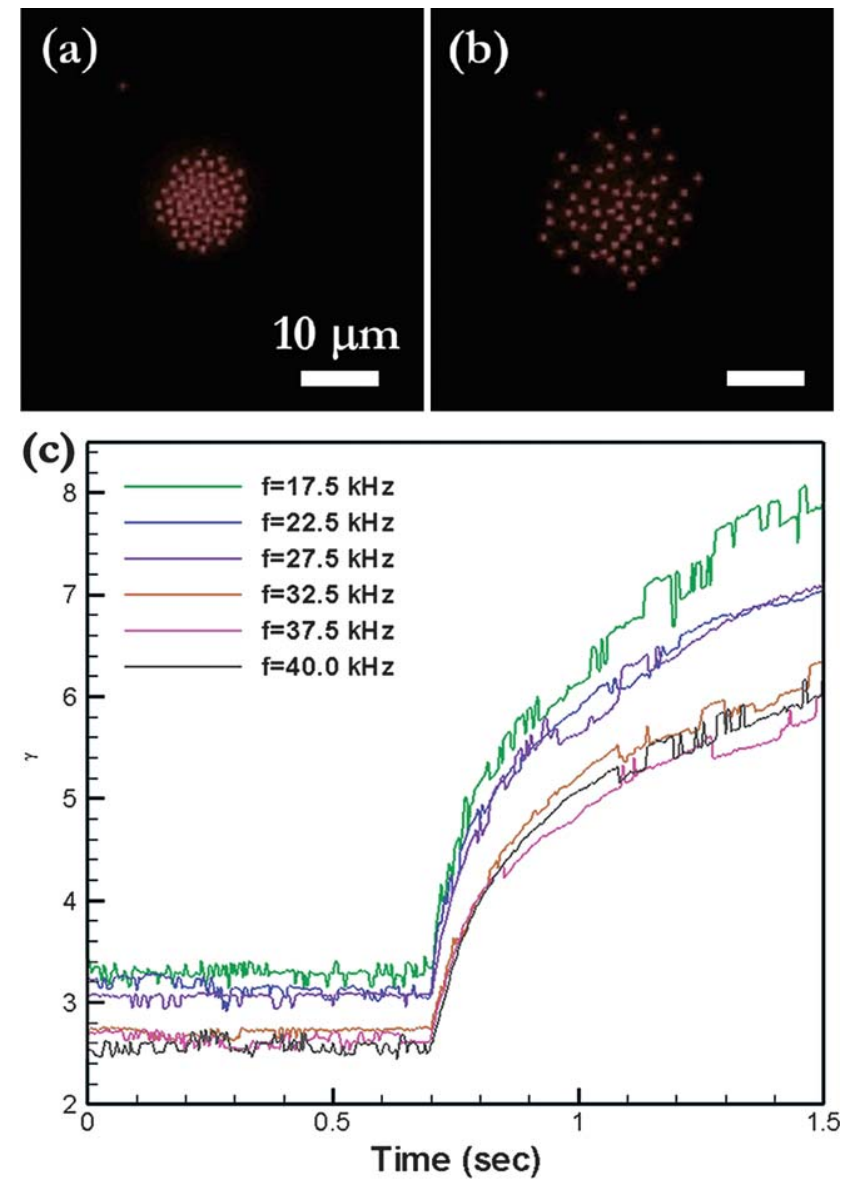

Fig. 7 Force spectroscopy based on REP. (a) A REP aggregation. (b) The aggregation 'explodes' when the laser is deactivated. (c) The time derivative of the ensemble averaged inter-particle distance provides a measure of the inter-particle force. Reprinted with permission from Kumar et al. ${ }^{70}$ Copyright 2010 American Chemical Society. the applied frequency. The physical basis for such sorting has been explained in terms of a critical AC frequency phenomenon. The critical AC frequency is the maximum AC frequency at which particles can be trapped. Kumar et al. ${ }^{70}$ used four different particle types and found that the critical AC frequency behaves as square of the particle radius $\left(\sim a^{2}\right)$ for a given particle type. Such a behavior is expected from non-equilibrium double-layer polarization theory. ${ }^{\mathbf{8 5}, 86,89}$ The sorting effect demonstrated by Williams et al. ${ }^{66}$ is the first sorting of colloids based on frequency dependent EDL polarization.

Apart from these applications REP has been extended to manipulate live bacteria, ${ }^{90}$ non-spherical particles ${ }^{91}$ and even nano-rods (unpublished data).

\subsection{Summary}

OEA, OET and REP appear distinct from each other both in original implementation and in their underlying principles. Hayward et al.'s ${ }^{13}$ setup seems to harness non-uniformities in current or charge distribution, whereas OET can create pronounced non-uniform electric fields mimicking traditional fabrication based electrokinetics. REP, in the setup employed by Williams et al., ${ }^{9}$ utilizes dominant ETH flows unlike the other techniques. However, these distinctions are hardly absolute; the techniques share some common operational principles. All these techniques use optics to induce gradient formation and both ITO and a-Si are photo-conductive, albeit to varying degrees. From an electrokinetics standpoint also there can be similarities. For example, REP in different renditions already utilizes $\mathrm{DEP}^{72}$ and/or $\mathrm{ACEO}^{92}$ to aid particle collection. Further research can only be expected to blur the lines between these schemes.

\section{Hybrid techniques with $l \approx 10^{-4}$ to $10^{-3} \mathrm{~m}$}

The previous section mainly deals with micron- and sub-micron sized objects. There exists another class of manipulation techniques dealing with droplets and the target length scales in this case range from $10^{-4}$ to $10^{-3} \mathrm{~m}$. Until now, OEW, FEOET, and some OEW derivatives are the major hybrid opto-electric techniques used for droplet handling. Their operating mechanisms, configurations, and performances will be discussed in the following content. Information regarding these techniques is also summarized in Table 2.

\subsection{Optoelectrowetting (OEW)}

Electrowetting (EW) is a technique well known for aqueous droplet manipulation by electrically driven surface tension and is a cornerstone in the field of digital microfluidics. The phenomenon was first explained by Gabriel Lippmann in 1875.93 According to his model, the contact angle of a drop of electrolyte on an electrode can be reduced by applying an electric potential across the electrolyte. The contact angle represents an equilibrium state of the surface tensions between the liquid, solid and gas phases. The external energy triggers the redistribution of the surface charges in both solid and liquid phases, which eventually reshapes the appearance of the aqueous droplet. Later, researchers ${ }^{6,93,94}$ came up with a modification, dubbed electrowetting on dielectric (EWOD), by adding a dielectric layer (e.g., 

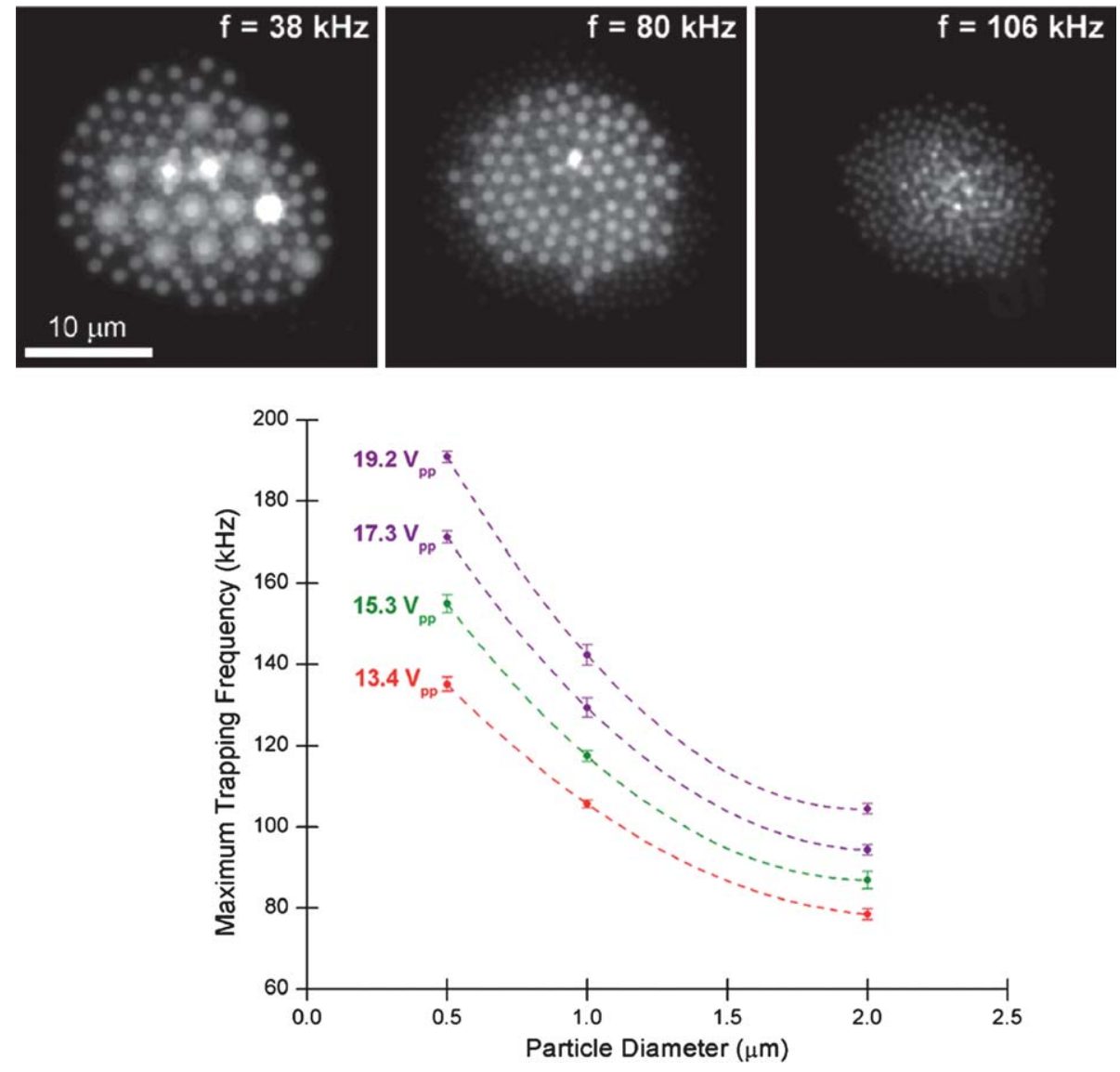

Fig. 8 Sorting with REP. $2.0 \mu \mathrm{m}, 1.0 \mu \mathrm{m}$, and $0.5 \mu \mathrm{m}$ polystyrene particles were all initially captured at $38 \mathrm{kHz} .2 .0 \mu \mathrm{m}$ particles were carried away at 80 $\mathrm{kHz}$. Only $0.5 \mu \mathrm{m}$ particles remained at $106 \mathrm{kHz}$. The maximum trapping frequency for particles tested. The fit is a second order polynomial, which is consistent with theory. Williams et al. ${ }^{66}$ Reproduced with permission from IOP publishing.

Table 2 Table listing the predominant hybrid techniques with $l \approx 10^{-4}$ to $10^{-3} \mathrm{~m}$

\begin{tabular}{llll}
\hline Technique & Configuration & Distinguishing features & Operating principle \\
\hline OEW & $\begin{array}{l}\text { Close chip, featureless electrodes, } \\
\text { and use of photoconductor }\end{array}$ & $\begin{array}{l}\text { Dynamic manipulation with virtual } \\
\text { electrodes }\end{array}$ & Electrowetting in an AC electric field \\
O-OEW & $\begin{array}{l}\text { Ond use of photoconductor } \\
\text { Dynamic manipulation with virtual } \\
\text { electrodes, and extensibility } \\
\text { and use of photoconductor }\end{array}$ & $\begin{array}{l}\text { Dynamic manipulation with virtual } \\
\text { electrodes, and ability to split } \\
\text { droplets }\end{array}$ & Electrowetting in an AC electric field \\
SCOEW & $\begin{array}{l}\text { Dynamic manipulation with virtual } \\
\text { electrodes }\end{array}$ & Electrowetting in a DC electric field \\
FEOET & and use of photoconductor & DEP in a DC electric field
\end{tabular}

$\mathrm{SiO}_{2}$ ) between the droplet and electrode to prevent electrolysis, and a hydrophobic coating (e.g., Teflon) on the top of the dielectric layer to lower hysteresis and increase contact angle. A hydrophobic coating is used to provide a restoring force to the droplet when the external potential is removed. The LippmannYoung equation is commonly used to show the relationship between the contact angle and applied potential:

$$
\cos \theta_{V}=\cos \theta_{0}+\frac{1}{2} \frac{\varepsilon_{0} \varepsilon_{\mathrm{r}}}{d \gamma_{\mathrm{lg}}} V^{2}
$$

where $\theta_{V}$ and $\theta_{0}$ are the contact angles, respectively, with and without potential, $\gamma_{\mathrm{lg}}$ is the gas/liquid surface tensions, $\varepsilon_{0}$ is the permittivity of vacuum, $\varepsilon_{\mathrm{r}}$ is the effective dielectric constant of the dielectric layer, $d$ is the thickness of the dielectric layer, and $V$ is the applied potential. Although EWOD has exhibited promising performance and proven itself a powerful tool for many applications, ${ }^{95-99}$ it still suffers from lack of flexibility, poor translational resolution, and incapability of simultaneously handling multiple droplets.

Optoelectrowetting (OEW) was first proposed by Chiou et al. ${ }^{10,100,101}$ to solve the deficiencies in the conventional electrowetting and enhance maneuverability. Lee et al. ${ }^{102}$ later reported an OEW device integrating with a $\mathrm{T}$-junction microchannel, to generate droplets and manipulate them in a row. 
The OEW configuration is based on EWOD scheme, with an additional photoconductive layer (Fig. 9a). A photoconductor intrinsically possesses low electrical conductivity, but when it is illuminated by a wavelength matching its energy gap, this conductivity increases substantially. In OEW, the
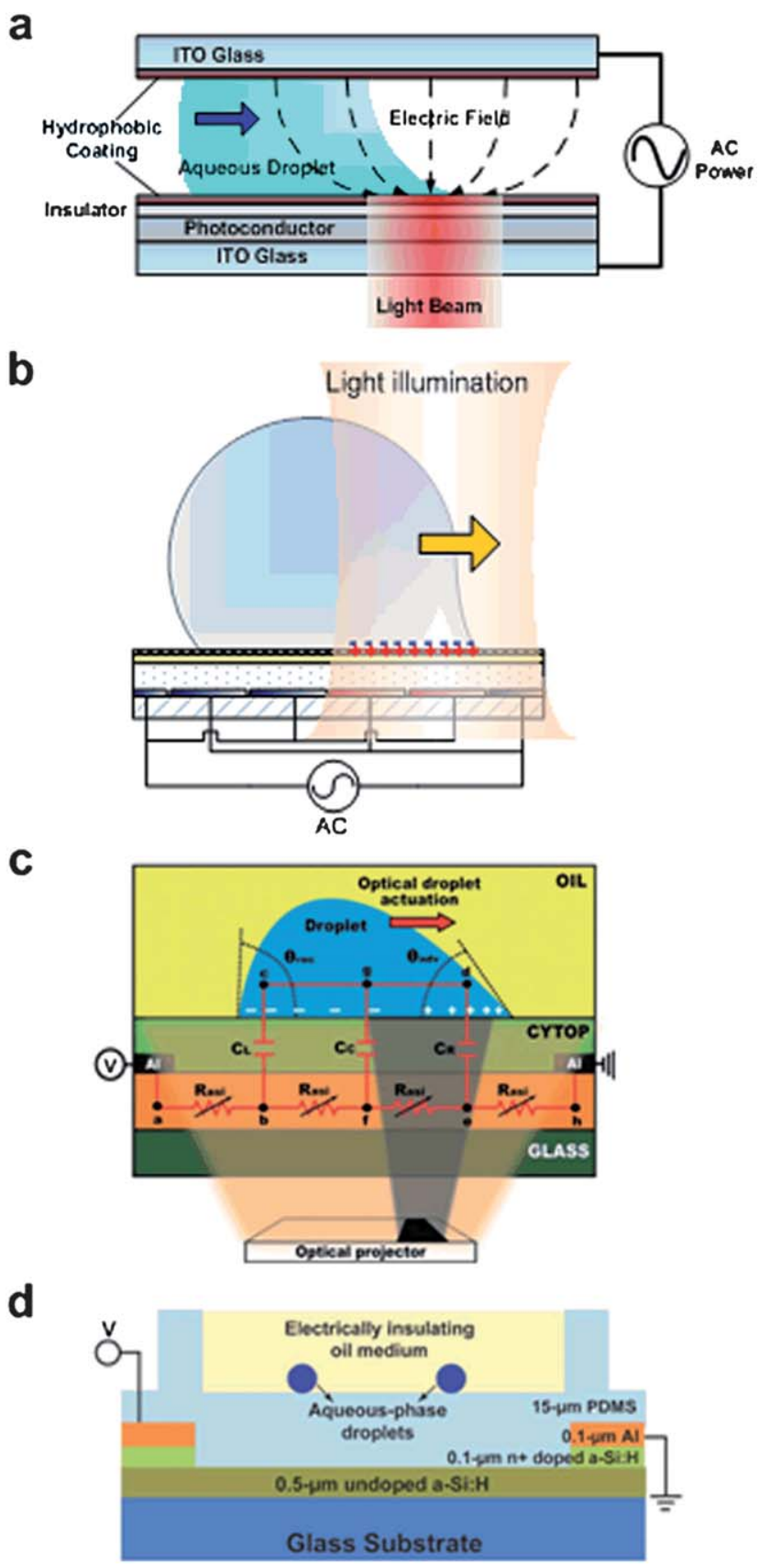

Fig. 9 Schematic of optoelectric droplet manipulation. (a) OEW, (b) OOEW. Except for the configuration, the mechanism of this technique is inherently the same as OEW. Reprinted with permission from Chuang et al. ${ }^{11}$ Copyright 2008, American Institute of Physics. (c) SCOEW. Unlike the other optoelectric counterparts, droplets on the device are driven by dark regions. Reprinted with permission from Park et al. ${ }^{12}$ Copyright 2010, Royal Society of Chemistry. (d) FEOET. Reprinted with permission from Park et al. ${ }^{103}$ Copyright 2008, American Institute of Physics. photoconductive layer is deployed between the insulator and electrode (ITO glass). When in the dark or in the absence of correct illumination, there is no wetting effect because the primary impedance drop occurs in the photoconductive layer. However, when illuminated by an appropriate light source, the major impedance drop switches to the insulating layer, causing the electrowetting phenomenon. A good photoconductive ratio (light-to-dark conductivity) relies on the relationship of the impedances of the photoconductor and the insulator. Specifically, the impedance of the photoconductor must be higher in the dark and lower in light than that of the insulator. Unlike conventional EW, OEW can only work under an alternating current (AC) regime due to the analogous RC circuit. Since an electrode pattern is virtually defined by the light source, programmable controls can be achieved with a featureless electrode layer. The capability of generating "virtual electrodes" imparts OEW the uniqueness of dynamic droplet manipulation.

Later Chuang et al. ${ }^{11}$ devised an open optoelectrowetting (O-OEW) technique by modifying the previous sandwiched OEW platform with coplanar interdigitated electrodes (Fig.9b). Droplet translational velocities, for a micro-litre droplet, as fast as $3.6 \mathrm{~mm} \mathrm{~s}^{-1}$ were measured. The equivalent circuit loops for O-OEW occur under a liquid droplet, instead of passing through the droplet as in a sandwiched device, thus removing the top cover for free space. Despite the difference in the structure, the driving mechanism appears to be the same as OEW. The open configuration endows the device with not only a higher degree of freedom, but also the flexibility of integration with additional devices. However, the electrode rearrangement lowers the efficiency. Recently Park et al. ${ }^{12}$ developed a unique droplet manipulation device, named singlesided continuous optoelectrowetting (SCOEW) (Fig. 9c) based on a different mechanism. A water droplet was immersed in oil to reduce hysteresis and increase contact angle. Speeds up to $102 \mathrm{~mm} \mathrm{~s}^{-1}$ on a $250 \mathrm{pL}$ droplet were recorded. In their design, a lateral electric field generated by a $1 \mathrm{kV}$ direct current (DC) bias was applied and the potential difference between the top and the bottom of the hydrophobic layer (used as an insulator as well) was used as the driving mechanism. The overall device can be modeled as an equivalent circuit composed of resistors and capacitors. Compared to O-OEW, the contact electrode pads in SCOEW are placed far apart on the surface of photoconductor and the manipulation area lies between them. Contrary to the previous OEW counterparts, the wetting surface is induced by a dark region. With equal illumination at both edges of a droplet or no illumination, there is no movement due to symmetric contact angle changes or no contact angle changes, respectively. However, on application of unequal illumination with a dark region inclined to one edge, the droplet is attracted to the dark side (i.e., the wetting surface) due to the asymmetry of the contact angles. A highlight of this technique is the capability of splitting a droplet utilizing liquid inertia (by a sudden application of a band of white light). However, operationally, SCOEW seems limited by the orientation of electric field. At least the current demonstration showed that droplets can only translate parallel to the electric field. This limitation restricts the potential development of the technique. 


\subsection{Floating electrode optoelectronic tweezers (FEOET)}

Similar to the concept of OET, floating electrode optoelectronic tweezers (FEOET) explores another frequency regime for aqueous droplet manipulation ${ }^{103,104}$ (Fig. 9d). Park et al. ${ }^{103,104}$ showed that a target object as large as a nano-litre droplet in a two-phase environment, such as water in oil, can be driven by a light-induced dipole moment. The electric field is created by a DC bias on two far separated electrodes. Under the electrodes is a layer of featureless photoconductor, while above the electrodes is a layer of PDMS housing the two-phase system. Based on the mechanism of DEP, the force can be affected by the volume of target $\left(V_{\mathrm{p}}\right)$, Clausius-Mossotti factor $\left(K_{\mathrm{CM}}\right)$, and electric field $(E)$ gradient:

$$
F_{\mathrm{DEP}}=\varepsilon_{0} \varepsilon_{\mathrm{m}} V_{\mathrm{p}} \operatorname{Re}\left[K_{\mathrm{CM}}\right] \nabla|\bar{E}|^{2}
$$

where $\varepsilon_{\mathrm{m}}$ is the effective dielectric constant of the surrounding medium. For a water-in-oil system, an aqueous droplet is more polarizable than oil, hence yielding positive DEP. Shining a light beam on the photoconductor creates a virtual electrode decreasing the surrounding electric field strength. Consequently, the droplet will be repelled from the illuminated spot when a light beam approaches the edge of the droplet. In contrast to OEW that usually works at lower electric potentials $\left(<100 \quad V_{\mathrm{pp}}\right)$, FEOET works at relatively high potentials $\left(>100 V_{\mathrm{dc}}\right)$ in order to induce sufficient forces to move a droplet. A maximum speed of $418.6 \mu \mathrm{m} \mathrm{s}^{-1}$, for a droplet $748 \mu \mathrm{m}$ in diameter, with a light intensity of $4.49 \mathrm{~mW} \mathrm{~mm}^{-2}$ was reported. Although, FEOET retains the advantages of the open configuration, from an operational point-of-view it inherently exerts far less force per unit than its electrowetting counterpart, OEW, which is driven by surface tension.

\subsection{Summary}

Hybrid techniques for droplet movement have borrowed heavily from existing EW schemes and the most important innovation has been in terms of the introduction of a photo-conductive layer. The concept of the 'virtual' electrodes provides a unifying theme to these different techniques, although the operational frequency domain, and hence the underlying physics, can be significantly different. The different device configurations that have been proposed allow these hybrid techniques to cater to diverse lab-on-a chip systems.

\section{Multi-scale tools $\left(l \approx 10^{-9}\right.$ to $\left.10^{-3} \mathrm{~m}\right)$}

The growth of digital microfluidic (i.e., droplet manipulation) platforms and its applications in sensor development and other areas are necessitating multi-scale manipulation strategies. This simultaneous need for control of droplet movement and manipulation of particulate phases inside droplets can be very challenging. Though limited progress has been achieved in this direction, ${ }^{92,105-107}$ hybrid tools with their higher degrees of freedom offer significant opportunities in this direction. For example Shah et al. ${ }^{107}$ demonstrated target separation and individual manipulation of HeLa cell samples by using a combination of EWOD and OET (Fig. 10a and b). In another work, taking advantage of O-OEW and integrating it with REP, a hybrid opto-electric device developed by Kumar et al. ${ }^{92}$ successfully demonstrated the potential for multi-scale manipulation (see Fig. 10c). In this integrated device, O-OEW is responsible for macro-scale droplet translation while REP is responsible for micro-scale particles handling. Overall an aqueous droplet containing particles is steered to the desired
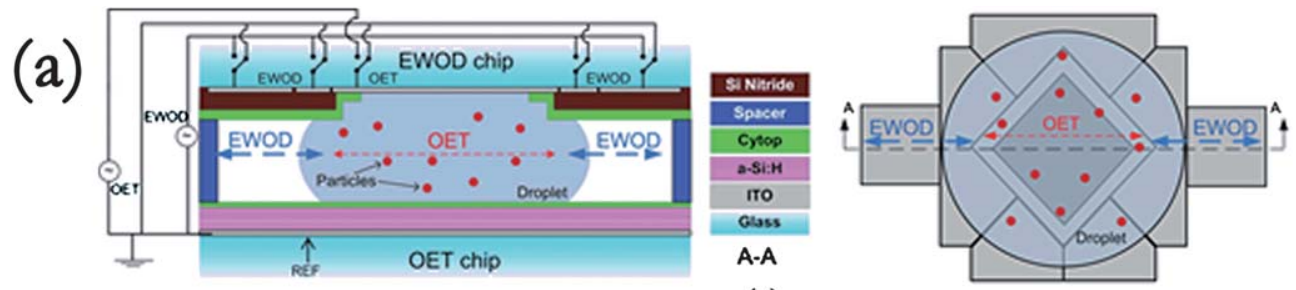

(a)
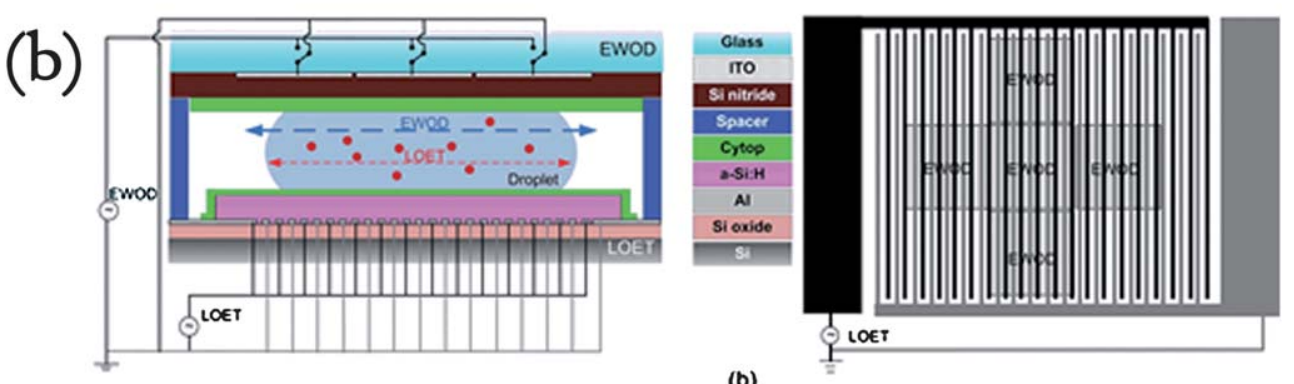

(b)

Fig. 10 (a) and (b) Two different multi-scale device configurations utilizing EWOD and OET. Shah et al. ${ }^{107}$ Reproduced by permission of the Royal Society of Chemistry. (c) An integrated optoelectric platform combining O-OEW and REP. The platform, composed of no microfluidic components though, is still capable of actively translating aqueous droplets and concentrating particles. Reprinted with permission from Kumar et al. ${ }^{92}$ Copyright 2010 American Chemical Society. 
work zone and then the inner particles are concentrated to enhance the signal. While, the tool developed by Kumar et al. ${ }^{92}$ has been portrayed as simple conjunction of O-OEW and REP, the underlying physical principles are significantly involved and more research is required in this direction.

These hybrid multi-scale techniques, which feature the ability to produce localized forces that can be scaled from millimetres to nanometres can potentially allow the development of novel, integrated sensing devices; preconcentrators that can collect and transport analytes to nano-sensors; micro-nano energy harvesting devices and nano-patterning of biological molecules.

We felt the need to discuss multi-scale manipulation tools as a separate section, as we believe that though in infancy, this has the potential to become a distinct field in itself. Its infancy also qualifies multi-scale tools to be listed in the section below.

\section{Opportunities and challenges}

History teaches us that tools like these usually undergo decades of development before they can be treated as mature. Just to provide a perspective, optical micromanipulation started in 1970s with the pioneering work of Ashkin, ${ }^{1}$ and the electrical counterpart also started out in the same decade when Herbert Pohl wrote his classic text. ${ }^{108}$ Even after close to 40 years of development, both these techniques are undergoing rigorous developments, with new findings and new insights being gained steadily. In comparison, hybrid opto-electric tools have just emerged and while they offer new opportunities, significant challenges remain. In this section, we will briefly describe the opportunities and challenges afforded by OET, REP, and OEW.

\subsection{Optoelectronic tweezers}

It has been demonstrated that OETs can manipulate, concentrate, and sort a variety of non-biological and biological particles. The most pertinent challenge with OETs, as is true with all of the opto-electric techniques mentioned herein, is to demonstrate a practical application of this technology. Recently, surface-enhanced Raman scattering (SERS) was incorporated with OETs for protein detection (lower limit of $0.1 \mathrm{ng} \mathrm{mL}^{-1}$ ) demonstrating its ability to detect antigens. ${ }^{109}$ As similar applications are developed, OETs will be accepted as a viable alternative. However, these applications cannot be developed until sample detection capabilities are integrated into such systems. The fabrication of OET platforms are straightforward, relying on illuminated photoconductive regions projected from one side of the platform to produce non-uniform fields in an otherwise uniform field region (refer Fig. 3). This arrangement restricts subsequent detection capabilities through optical means, accessing the region of interest through the top transparent ITO substrate. If additional non-optical sensing elements like microelectrodes are introduced onto the platform these can interfere with the otherwise uniform field, generating unwanted nonuniform field electrokinetics. The demonstration of simultaneous particle manipulation and detection is a current challenge in OET.

The value in OET systems is its inherent ability to dynamically create 'virtual' electrodes that are reconfigurable and can span a large working area on the device.
The same photoconductive platform can manipulate droplets through OEW, and a combination of bulk fluid handling with micro-bead sorting makes this technique valuable. Therefore it can be envisioned that a photoconductive platform can be readily programmed at the user's discretion, enabling multiple uses for a single device. This multipurpose capability has added value. However, this technology is in direct competition with a variety of single-use, single-purpose microfluidic chips that do not rely on either optic or electrokinetic-based fluid handling. A demonstration of a variety of practical applications being performed on the same platform, though, is necessary to draw additional attention to this technique.

\subsection{Rapid electrokinetic patterning}

In its simplest rendition, REP utilizes a sandwiched microfluidic setup, along with a source of optical landscapes. While the optics hardware can be intricate, such an infrastructure already exists in the form of a prevalent optical tweezers setup. An optical tweezer setup operating at the correct wavelength can easily be the source of optical landscapes involved in REP. Thus, replicating REP in an existing optical tweezer laboratory is a relatively straightforward possibility. Moreover, since the requirements of REP on the illuminating beam are not as stringent (example: one can use low N.A. lenses with REP), a significant opportunity exists in the development of low cost portable REP systems.

Applications of REP are at present in its nascent stage, however opportunities exist both from an applied and fundamental electrokinetics perspective. Integration with an existing micro-total-analytical system ( $\mu$ TAS) setup and use of REP for sample pre-concentration processes can be one avenue of exploration. REP with its collection of analytes from the bulk can be used to enhance the performance of microfluidic sensors. The use of electrothermal vortices in REP can also be utilized in the form of enhanced mixing for assays, or even possibly give a dynamic nature to existing electrothermal pumping schemes. ${ }^{110,111}$ Moreover, REP need not be confined to simple electrode geometries as we have seen earlier. ${ }^{72,92}$ As exploration of REP in complex electrode geometries has been rather limited, considerable opportunity exists in that direction also.

REP allows a unique decoupling of electrokinetic mechanisms such as ACEO from ETH flows, flows from particle-particle interactions. Hence from an electrokinetics perspective, REP provides a unique opportunity to explore and characterize some of these fundamental forces.

\subsection{Optoelectrowetting and floating electrode optoelectronic tweezers}

Microfluidics plays an intermediate role in bridging our macroscopic world and nanoscopic territory. Therefore, the fundamental requirements, such as interfacing, maneuverability, and throughput, constantly pose challenges to the scientific community. Digital microfluidics (i.e., droplet manipulation) as a rapidly growing research field among the broad microfluidic spectrum provides a unique alternative in liquid handling. The potential programmability of digital microfluidics further consolidates the idea of lab-in-a-drop. ${ }^{112-115}$ Although tremendous research work has been achieved in this area, OEW still 
draws much attention due to its excellent capability of dynamic manipulation and massively parallel processing. The subsequent derivatives, such as O-OEW, SCOEW, or FEOET, offer easy integration with other technologies as well as more flexible manipulation. The subtle combination of both electrowetting and photoeletronic elements provides a niche in the emerging lab-on-a-chip territory.

Inkjet printing, pharmacy, and point-of-care biochips are several well-known mature markets that have been running for years with different droplet processing techniques. Apparently the development of OEW has fueled some special interests from the existing markets. The concept of lab-on-a-chip can be further carried out by this technique. In continuous microfluidics regime, various microfluidic components, such as valves, pumps, reservoirs, channels, etc., must be fabricated and connected deliberately to perform a general-purpose platform. ${ }^{116}$ In this case, the liquid fluids are driven and controlled by numerous pneumatic valves. The sequential procedures are coordinated by computerbased programs. In digital microfluidics, however, the same task can be easily achieved in the form of lab-in-a-drop. The ability to integrate with other techniques and the programmability not only differentiates OEW from other counterparts, but also make it a promising tool in microfluidics.

\section{Discussion and future prospects}

Hybrid opto-electric techniques are the result of an extraordinary synergy of optical and electrical energy. These progeny of optical and electrical systems inherit many of the good qualities of their parents and thus allow dynamic, multi-scale and highthroughput manipulation. While still at a developing stage these hybrid techniques have opened new portals to fundamental sciences at the diminutive length scales as well as have generated a collection of new applications. These technologies have been very well received by the scientific community; ${ }^{65,117}$ however in order to stand the test of time, it is essential that new researchers bring in fresh ideas into this field and enrich the progress that has already been made. Time and again in science, new minds have brought in unconventional applications to existing tools, and while some hybrid techniques like OET are in this process, techniques like REP and OEA could benefit enormously from a new look.

\section{Acknowledgements}

A. Kumar performed the work as a Eugene P. Wigner Fellow and staff member at the Oak Ridge National Laboratory, managed by UT-Battelle, LLC, for the U.S. Department of Energy under Contract DE-AC05-00OR22725.

\section{References}

1 A. Ashkin, Phys. Rev. Lett., 1970, 24, 156.

2 A. Ashkin, J. M. Dziedzic and T. Yamane, Nature, 1987, 330, 769771.

3 H. Morgan and N. G. Green, AC Electrokinetics: Colloids and Nanoparticles, Research Studies Press, Philadelphia, PA, 2003.

4 T. B. Jones, Electromechanics of Particles, Cambridge University Press, New York, 1995.

5 F. Petersson, L. Aberg, A. M. Sward-Nilsson and T. Laurell, Anal. Chem., 2007, 79, 5117-5123.
6 M. G. Pollack, A. D. Shenderov and R. B. Fair, Lab Chip, 2002, 2, 96-101.

7 A. Ashkin, Proc. Natl. Acad. Sci. U. S. A., 1997, 94, 4853-4860.

8 P. Y. Chiou, A. T. Ohta and M. C. Wu, Nature, 2005, 436, 370-372.

9 S. J. Williams, A. Kumar and S. T. Wereley, Lab Chip, 2008, 8, 1879 1882.

10 P. Y. Chiou, H. Moon, H. Toshiyoshi, C. J. Kim and M. C. Wu, Sens. Actuators, A, 2003, 104, 222-228.

11 H. S. Chuang, A. Kumar and S. T. Wereley, Appl. Phys. Lett., 2008, 93, 064104

12 S. Y. Park, M. A. Teitell and E. P. Y. Chiou, Lab Chip, 2010, 10, $1655-1661$.

13 R. C. Hayward, D. A. Saville and I. A. Aksay, Nature, 2000, 404, 56-59.

14 T. Y. Gong and D. W. M. Marr, Appl. Phys. Lett., 2004, 85, 3760 3762.

15 M. Bohmer, Langmuir, 1996, 12, 5747-5750.

16 P. Richetti, J. Prost and P. Barois, J. Phys., Lett., 1984, 45, 11371143.

17 M. Trau, D. A. Saville and I. A. Aksay, Science, 1996, 272, 706-709.

18 M. Trau, D. A. Saville and I. A. Aksay, Langmuir, 1997, 13, 63756381.

19 M. Z. Bazant and T. M. Squires, Phys. Rev. Lett., 2004, 92, 066101.

20 T. M. Squires, Lab Chip, 2009, 9, 2477-2483.

21 T. M. Squires and M. Z. Bazant, J. Fluid Mech., 2004, 509, $217-$ 252.

22 W. D. Ristenpart, I. A. Aksay and D. A. Saville, Phys. Rev. E: Stat. Phys., Plasmas, Fluids, Relat. Interdiscip. Top., 2004, 69, 021405.

23 Y. Solomentsev, M. Bohmer and J. L. Anderson, Langmuir, 1997, 13, 6058-6068.

24 Y. Solomentsev, S. A. Guelcher, M. Bevan and J. L. Anderson, Langmuir, 2000, 16, 9208-9216.

25 W. D. Ristenpart, I. A. Aksay and D. A. Saville, J. Fluid Mech., 2007, 575, 83-109.

26 K. H. Bhatt, S. Grego and O. D. Velev, Langmuir, 2005, 21, $6603-$ 6612.

27 V. Brisson and R. D. Tilton, Biotechnol. Bioeng., 2002, 77, 290-295.

28 W. M. Choi and O. Park, Nanotechnology, 2006, 17, 325-329.

29 R. K. Golding, P. C. Lewis and E. Kumacheva, Langmuir, 2004, 20, 1414-1419.

30 F. Nadal, F. Argoul, P. Hanusse, B. Pouligny and A. Ajdari, Phys. Rev. E: Stat. Phys., Plasmas, Fluids, Relat. Interdiscip. Top., 2002, 65, 061409 .

31 W. D. Ristenpart, I. A. Aksay and D. A. Saville, Phys. Rev. Lett., 2003, 90, 128303.

32 W. D. Ristenpart, I. A. Aksay and D. A. Saville, Langmuir, 2007, 23, 4071-4080.

33 W. D. Ristenpart, P. Jiang, M. A. Slowik, C. Punckt, D. A. Saville and I. A. Aksay, Langmuir, 2008, 24, 12172-12180.

34 W. D. Ristenpart, P. G. Kim, C. Domingues, J. Wan and H. A. Stone, Phys. Rev. Lett., 2007, 99, 234502.

35 S. R. Yeh, M. Seul and B. I. Shraiman, Nature, 1997, 386, 57-59.

36 P. Y. Chiou, Z. H. Chang and M. C. Wu, 2003 Ieee/Leos International Conference on Optical Mems, 2003, pp. 8-9.

37 J. H. Wei and S. C. Lee, J. Appl. Phys., 1993, 76, 1033-1040.

38 S. Adachi, Optical Properties of Crystalline and Amorphous Semiconductors: Materials and Fundamental Principles, Kluwer Academic Publishers, Boston, 1999.

39 P. Y. Chiou, A. T. Ohta, A. Jamshidi, H. Y. Hsu and M. C. Wu, J. Microelectromech. Syst., 2008, 17, 525-531.

40 W. Choi, S. H. Kim, J. Jang and J. K. Park, Microfluid. Nanofluid., 2007, 3, 217-225.

41 H. Hwang, Y. J. Choi, W. Choi, S. H. Kim, J. Jang and J. K. Park, Electrophoresis, 2008, 29, 1203-1212.

42 Y. S. Lu, Y. P. Huang, J. A. Yeh, C. Lee and Y. H. Chang, Opt. Quantum Electron., 2005, 37, 1385-1395.

43 H. Hwang, Y. Oh, J. J. Kim, W. Choi, J. K. Park, S. H. Kim and J. Jang, Appl. Phys. Lett., 2008, 92, 024108-024110.

44 A. T. Ohta, P. Y. Chiou, H. L. Phan, S. W. Sherwood, J. M. Yang, A. N. K. Lau, H. Y. Hsu, A. Jamshidi and M. C. Wu, IEEE J. Sel. Top. Quantum Electron., 2007, 13, 235-243.

45 G. Fuhr, T. Mueller, V. Baukloh and K. Lucas, Hum. Reprod., 1998, 13, 136-141.

46 H. Y. Hsu, A. T. Ohta, P. Y. Chiou, A. Jamshidi, S. L. Neale and M. C. Wu, Lab Chip, 2010, 10, 165-172. 
47 H. Y. Hsu, A. T. Ohta, P. Y. Chiou, A. Jamshidi and M. C. Wu, Phototransistor-based Optoelectronic Tweezers for Cell Manipulation in Highly Conductive Solution, Lyon, France, 2007.

48 J. K. Valley, A. Jamshidi, A. T. Ohta, H. Y. Hsu and M. C. Wu, J. Microelectromech. Syst., 2008, 17, 342-350.

49 A. Jamshidi, P. J. Pauzauskie, P. J. Schuck, A. T. Ohta, P. Y. Chiou, J. Chou, P. D. Yang and M. C. Wu, Nat. Photonics, 2008, 2, 85-89.

50 W. Choi, S. W. Nam, H. Hwang, S. Park and J. K. Park, Appl. Phys. Lett., 2008, 93, 143901.

51 H. Hwang, J. J. Kim and J. K. Park, J. Phys. Chem. B, 2008, 112, 9903-9908.

52 A. Ramos, H. Morgan, N. G. Green and A. Castellanos, J. Colloid Interface Sci., 1999, 217, 420-422.

53 H. Hwang and J. K. Park, Lab Chip, 2009, 9, 199-206.

54 N. G. Green, A. Ramos, A. Gonzalez, A. Castellanos and H. Morgan, J. Phys. D: Appl. Phys., 2000, 33, L13-L17.

55 A. Jamshidi, S. L. Neale, K. Yu, P. J. Pauzauskie, P. J. Schuck, J. K. Valley, H. Y. Hsu, A. T. Ohta and M. C. Wu, Nano Lett., 2009, 9, 2921-2925.

56 M. W. Lee, Y. H. Lin and G. B. Lee, Microfluid. Nanofluid., 2010, 8, 609-617.

57 A. T. Ohta, M. Garcia, J. K. Valley, L. Banie, H. Y. Hsu, A. Jamshidi, S. L. Neale, T. Lue and M. C. Wu, Lab Chip, 2010, 10, 3213-3217.

58 A. T. Ohta, P. Y. Chiou, T. H. Han, J. C. Liao, U. Bhardwaj, E. R. B. McCabe, F. Q. Yu, R. Sun and M. C. Wu, J. Microelectromech. Syst., 2007, 16, 491-499.

59 H. Hwang, D. H. Lee, W. J. Choi and J. K. Park, Biomicrofluidics, 2009, 3, 014103.

60 J. K. Valley, S. Neale, H. Y. Hsu, A. T. Ohta, A. Jamshidi and M. C. Wu, Lab Chip, 2009, 9, 1714-1720.

61 P. Y. Chiou, W. H. Wong, J. C. Liao and M. C. Wu, Presented in Part at the Proceedings of the 17th IEEE International Conference on Micro Electro Mechanical Systems (MEMS), Maastricht, Netherlands, 2004.

62 M. Hoeb, J. O. Radler, S. Klein, M. Stutzmann and M. S. Brandt, Biophys. J., 2007, 93, 1032-1038.

63 S. M. Yang, T. M. Yu, H. P. Huang, M. Y. Ku, L. Hsu and C. H. Liu, Opt. Lett., 2010, 35, 1959-1961.

64 A. N. K. Lau, A. T. Ohta, H. L. Phan, H. Y. Hsu, A. Jamshidi, P. Y. Chiou and M. C. Wu, Lab Chip, 2009, 9, 2952-2957.

65 H. Hwang and J. K. Park, Lab Chip, 2011, 11, 33-47.

66 S. J. Williams, A. Kumar, N. G. Green and S. T. Wereley, $J$ Micromech. Microeng., 2010, 20, 015022.

67 S. J. Williams, A. Kumar, N. G. Green and S. T. Wereley, Nanoscale, 2009, 1, 133-137.

68 S. J. Williams, A. Kumar, S. T. Wereley, Optically induced electrokinetic patterning and manipulation of particles, http://hdl handle.net/1813/11399.

69 S. J. Williams, A. Kumar and S. T. Wereley, Phys. Fluids, 2009, 21, 091104.

70 A. Kumar, J.-S. Kwon, S. J. Williams, N. G. Green, N. K. Yip and S. T. Wereley, Langmuir, 2010, 26, 5262-5272.

71 N. G. Green, A. Ramos, A. Gonzalez, A. Castellanos and H. Morgan, J. Electrost., 2001, 53, 71-87.

72 A. Kumar, S. J. Williams and S. T. Wereley, Microfluid. Nanofluid., $2009,6,637-646$

73 A. Kumar, C. Cierpka, S. Williams, C. Kähler and S. Wereley, Microfluid. Nanofluid., 2011, 10, 355-365.

74 C. Cierpka, R. Segura, R. Hain and C. J. Kahler, Meas. Sci. Technol., 2010, 21, 045401.

75 J. A. Fagan, P. J. Sides and D. C. Prieve, Langmuir, 2004, 20, 48234834.

76 J. A. Fagan, P. J. Sides and D. C. Prieve, Langmuir, 2005, 21, 1784 1794.

77 J. A. Fagan, P. J. Sides and P. C. Prieve, Langmuir, 2002, 18, 7810-7820.

78 M. M. Hatlo and L. Lue, Soft Matter, 2008, 4, 1582-1596.

79 J. Lyklema, H. P. V. Leeuwen, M. V. Vliet and A. M. Cazabat, Fundamentals of Interface and Colloid Science, Academic Press, London; San Diego, 1991.

80 S. Chakraborty, in Encyclopedia of Microfluidics and Nanofluidics, ed. D. Li, Springer, Berlin, 2008, vol. I.
81 S. Chakraborty and A. K. Srivastava, Langmuir, 2007, 23, 1242112428.

82 P. K. Das and S. Bhattacharjee, Langmuir, 2005, 21, 4755-4764

83 S. S. Dukhin, Adv. Colloid Interface Sci., 1993, 44, 1-134.

84 J. M. Schurr, J. Phys. Chem., 1964, 68, 2407.

85 H. P. Schwan, G. Schwarz, J. Maczuk and H. Pauly, J. Phys. Chem., 1962, 66, 2626

86 G. Schwarz, J. Phys. Chem., 1962, 66, 2636.

87 M. Fixman, J. Chem. Phys., 1983, 78, 1483-1491.

88 N. G. Green and H. Morgan, J. Phys. Chem. B, 1999, 103, 41-50.

89 C. T. O'Konski, J. Phys. Chem., 1960, 64, 605-619.

90 S. T. Wereley, A. Kumar, J.-S. Kwon, S. Ravindranath and J. Irudayaraj, Presented in Part at the IMECE 2010: Proceedings of the Asme International Mechanical Engineering Congress and Exposition, Vancouver, Canada, November 12-18, 2010.

$91 \mathrm{R}$. V. Thakur and S. T. Wereley, Presented in Part at the IMECE 2010: Proceedings of the Asme International Mechanical Engineering Congress and Exposition, Vancouver, Canada, November 12-18, 2010.

92 A. Kumar, H. S. Chuang and S. T. Wereley, Langmuir, 2010, 26, 7656.

93 G. Lippmann, Ann. Chim. Phys., 1875, 5, 494-549.

94 S. K. Cho, S. K. Fan, H. Moon and C. J. Kim, Toward Digital Microfluidic Circuits: Creating, Transporting, Cutting and Merging Liquid Droplets by Electrowetting-Based Actuation, Las Vegas, NV, 2002.

95 I. Moon and J. Kim, Sens. Actuators, A, 2006, 130-131, 537-544.

96 P. Paik, V. K. Pamula, M. G. Pollack and R. B. Fair, Lab Chip, 2003, 3, 28-33.

97 V. Srinivasan, V. K. Pamula and R. B. Fair, Anal. Chim. Acta, 2004, 507, 145-150.

98 A. R. Wheeler, H. Moon, C. J. Kim, J. A. Loo and R. L. Garrell, Anal. Chem., 2004, 76, 4833-4838.

99 J. Y. Yoon and R. L. Garrell, Anal. Chem., 2003, 75, 5097-5102.

100 P. Y. Chiou, Z. Chang and M. C. Wu, J. Microelectromech. Syst., 2008, 17, 133-138.

101 P. Y. Chiou and M. C. Wu, Presented in Part at the Solid-State Sensor, Actuator and Microsystems Workshop, Hilton Head Island, South Carolina, June 2-6, 2002.

102 D.-H. Lee, H. Hwang and J.-K. Parka, Appl. Phys. Lett., 2009, 95, 164102.

103 S. Y. Park, C. Pan, T.-H. Wu, C. Kloss, S. Kalim, C. E. Callahan, M. Teitell and E. P. Y. Chiou, Appl. Phys. Lett., 2008, 92, 151101.

104 S.-Y. Park, S. Kalim, C. Callahan, M. A. Teitell and E. P. Y. Chiou, Lab Chip, 2009, 9, 3228-3235.

105 S. K. Cho, Y. J. Zhao and C. J. Kim, Lab Chip, 2007, 7, 490-498.

106 S. K. Fan, P. W. Huang, T. T. Wang and Y. H. Peng, Lab Chip, $2008,8,1325-1331$.

107 G. J. Shah, A. T. Ohta, E. P. Y. Chiou, M. C. Wu and C. J. Kim, Lab Chip, 2009, 9, 1732-1739.

108 H. A. Pohl, Dielectrophoresis, Cambridge Univ. Press, Cambridge, UK, 1978.

109 H. Hwang, H. Chon, J. Choo and J. K. Park, Anal. Chem., 2010, 82, $7603-7610$

110 M. Lian, N. Islam and J. Wu, IET Nanobiotechnol., 2007, 1, 3643.

111 M. Lian and J. Wu, Appl. Phys. Lett., 2009, 94, 064101.

112 J. Clausell-Tormos, D. Lieber, J.-C. Baret, A. El-Harrak, O. J. Miller, L. Frenz, J. Blouwolff, K. J. Humphry, S. Koster, H. Duan, C. Holtze, D. A. Weitz, A. D. Griffiths and C. A. Merten, Chem. Biol., 2008, 15, 427-437.

113 S. Koster, F. E. Angile, H. Duan, J. J. Agresti, A. Wintner, C. Schmitz, A. C. Rowat, C. A. Merten, D. Pisignano, A. D. Griffithsc and D. A. Weitz, Lab Chip, 2008, 8, 1110-1115.

114 J. Pipper, Y. Zhang, P. Neuzil and T.-M. Hsieh, Angew. Chem., Int. Ed., 2008, 47, 3900-3904.

115 A. Sukhanova, Y. Volkov, A. L. Rogach, A. V. Baranov, A. S. Susha, D. Klinov, V. Oleinikov, J. H. M. Cohen and I. Nabiev, Nanotechnology, 2007, 18, 185602.

116 J. W. Hong and S. R. Quake, Nat. Biotechnol., 2003, 21, 1179-1183.

117 S. Choi, M. Goryll, L. Sin, P. Wong and J. Chae, Microfluid. Nanofluid., 2010, 1-17. 